\title{
Oral squamous cell carcinoma may originate from bone marrow-derived stem cells
}

\author{
TOMONORI HASEGAWA ${ }^{1}$, KOH-ICHI NAKASHIRO ${ }^{2}$, CHONJI FUKUMOTO $^{1}$, TOSHIKI HYODO ${ }^{1}$, \\ YUTA SAWATANI $^{1}$, MICHIKO SHIMURA ${ }^{1}$, RYOUTA KAMIMURA ${ }^{1}$, NOBUYUKI KURIBAYASHI ${ }^{2}$, \\ ATSUSHI FUJITA ${ }^{1}$, DAISUKE UCHIDA ${ }^{2}$ and HITOSHI KAWAMATA ${ }^{1}$ \\ ${ }^{1}$ Department of Oral and Maxillofacial Surgery, Dokkyo Medical University School of Medicine, \\ Tochigi 321-0293; ${ }^{2}$ Department of Oral and Maxillofacial Surgery, \\ Ehime University Graduate School of Medicine, Ehime 791-0295, Japan
}

Received July 16, 2020; Accepted October 21, 2020

DOI: $10.3892 / \mathrm{ol} .2021 .12431$

\begin{abstract}
Molecules that demonstrate a clear association with the aggressiveness of oral squamous cell carcinoma (OSCC) have not yet been identified. The current study hypothesized that tumor cells in OSCC have three different origins: Epithelial stem cells, oral tissue stem cells from the salivary gland and bone marrow (BM) stem cells. It was also hypothesized that carcinomas derived from less-differentiated stem cells have a greater malignancy. In the present study, sex chromosome analysis by fluorescence in situ hybridization and/or microdissection PCR was performed in patients with OSCC that developed after hematopoietic stem cell transplantation (HSCT) from the opposite sex. OSCC from 3 male patients among the 6 total transplanted patients were considered to originate from donor-derived BM cells. A total of 2/3 patients had distant metastasis, resulting in a poor prognosis. In a female patient with oral potentially malignant disorder who underwent HSCT, there were $10.7 \%$ Y-containing cells in epithelial cells, suggesting that some epithelial cells were from the donor. Subsequently, gene expression patterns in patients with possible BM stem cell-derived OSCC were compared with those in patients with normally developed OSCC by microarray analysis. A total of 3 patients with BM stem cell-derived OSCC exhibited a specific pattern of gene expression. Following cluster analysis by the probes identified on BM stem cell-derived OSCC, 2 patients with normally developed OSCC were included in the cluster of BM stem cell-derived
\end{abstract}

Correspondence to: Dr Hitoshi Kawamata, Department of Oral and Maxillofacial Surgery, Dokkyo Medical University School of Medicine, 880 Kitakobayashi, Mibu, Shimo-tsuga, Tochigi 321-0293, Japan

E-mail: h-kawama@dokkyomed.ac.jp

Key words: oral squamous cell carcinoma, fluorescence in-situ hybridization analysis, microarray analysis, clustering analysis, bone marrow derived stem cells, sex chromosome, mesenchymal stem cells, hematopoietic stem cell transplantation
OSCC. If the genes that could discriminate the origin of OSCC were identified, OSCCs were classified into the three aforementioned categories. If diagnosis can be performed based on the origin of the cancer cells, a more specific therapeutic strategy may be implemented to improve prognosis. This would be a paradigm shift in diagnostic and therapeutic strategies for OSCC.

\section{Introduction}

Oral squamous cell carcinoma (OSCC) is highly invasive locally and often leads to cervical lymph node metastasis, with distant metastasis being less frequent $(1,2)$. Factors related to these properties of OSCC $(3,4)$ include oncogene products (5-9), mutated tumor suppressor gene products (10-12), adhesion molecules (13-15), matrix metalloproteinases (16-18) and cell cycle proteins (19-21). However, a molecule clearly associated with the aggressiveness of OSCC has not yet been identified. Similarly, histopathological features can be linked to superficial properties of OSCC, but cannot specifically explain its invasive and metastatic potentials (22). Given this background, we hypothesized that the identification of the origin of the tumor or tumor stem cells may be important in predicting the aggressiveness of OSCC.

Following advances in regenerative medicine, several somatic stem cells have been identified and the importance in regenerating certain organs has been recognized. It has been reported that hepatic and endothelial stem cells are derived from the bone marrow (BM) and are present in circulating blood $(23,24)$. Furthermore, BM stem cells may be involved in the regeneration of the gastrointestinal epithelium (25), and gastric cancer can develop from these cells (26). Tamai et al found that BM stem cells (mesenchymal stem cells) are involved in epithelial repair of skin lesions in patients with hereditary epidermolysis bullosa (27). It is well known that BM stem cells include hematopoietic and mesenchymal stem cells. Multilineage-differentiating stress-enduring (Muse) cells, a small population of BM mesenchymal stem cells in circulating blood, can also differentiate into several types of cells, including epithelial cells (28). 
The present study attempted to establish a novel diagnostic criterion and a method that can determine the fundamental malignancy of the tumor cells without being confused by masquerade images or minor genetic abnormalities. The present study was based on the hypothesis that there are three origins of tumor cells in OSCC: Epithelial stem cells, oral tissue stem cells from the salivary gland and BM stem cells. We also hypothesized that carcinoma derived from less-differentiated stem cells has a higher malignancy. It was shown that OSCC derived from stem cells in the salivary gland had a significantly higher metastatic potential and poorer prognosis among oral carcinomas histopathologically diagnosed as SCC (29). In addition, a case of a male patient who underwent hematopoietic stem cell transplantation (HSCT) from his sister for treating leukemia, and developed an oral lichenoid lesion due to chronic graft versus host disease following HSCT was reported. It was demonstrated that oral lichenoid lesion epithelial cells from the patient originated from donor BM stem cells, and these cells developed SCC on the mandibular gingiva (30).

In the present study, sex chromosome analysis was performed in patients with OSCC developed following HSCT from the opposite gender, in order to examine whether OSCC originates from BM stem cells. Gene expression patterns in patients with possibly BM stem cell-derived OSCC were compared with those in patients with normally developed OSCC by microarray analysis, in order to examine whether OSCC with a possible BM origin shows a specific pattern of the gene expression.

\section{Patients and methods}

\section{Patients}

Sex chromosome analysis. Six patients (5 males and 1 female) who developed graft versus host disease (GvHD) in the oral mucosa following HSCT from a donor of the opposite sex and then developed OSCC were examined by fluorescence in situ hybridization (FISH) and G-band staining of sex chromosomes (Tables I and II). The patients (cases 1 to 6) all presented with a lichenoid lesion, a symptom of chronic GvHD, in the oral mucosa and subsequently developed OSCC 5 to 18 years after HSCT. All patients underwent surgery for OSCC and 4 patients (cases 1, 2, 3 and 6) died from primary disease or multiple metastases. Case 7 was a male patient with OSCC who did not undergo HSCT. Three patients (cases 8, 9, 10; 2 males, 1 female) presented with a lichenoid lesion in the oral mucosa following HSCT from an opposite-sex donor, and were also examined by sex chromosome FISH (Tables I and II). Two male patients with normal labial mucosa, who did not undergo HSCT, were also subjected to this analysis (Table I). All of the above patients were treated between 2006 and 2018 at the Dokkyo University (Mibu, Japan) or Ehime University Hospitals (Toon, Japan).

Assessment by microarray analysis. A total of 16 samples from 14 patients with oral SCC (11 men and 3 women) were subjected to microarray analysis. One patient (case 3 ) had two independent tumors in the oral cavity. Two samples from different part of 1 tumor of 1 patient (case 17) was also analyzed. Each tumor was surgically resected at the Dokkyo
Medical University and Ehime University Hospitals between 2017 and 2018. None of the patients had received previous radiotherapy or chemotherapy. The clinical characteristics of these patients are shown in Table III.

Histopathological examination. Tissue samples were fixed in formalin and embedded in paraffin. Sections of $4-\mu \mathrm{m}$ were stained with hematoxylin and eosin, and reviewed by experienced pathologists for histopathological diagnosis. Tumor cell differentiation (WHO classification) (31), Anneroth grade (32), Y-K mode of invasion (22) and pathological lymph node metastasis were assessed. The histopathologic features of all patients in the study are shown in Tables I and III.

FISH analysis. Sections $(5-\mu \mathrm{m})$ were deparaffinized and treated with $0.5 \%$ pepsin solution and $0.1 \mathrm{~N} \mathrm{HCl}$ at $37^{\circ} \mathrm{C}$ for 12-19 min. Following neutralization with PBS, the solution was washed with distilled water and dried. Dig-labeled human $\mathrm{X}$ chromosome and FITC-labeled Y chromosome (both from Chromosome Science Labo, Inc., Hokkaido, Japan) probe solutions were added, and the sections and probes were simultaneously denatured on a hot plate at $90^{\circ} \mathrm{C}$ for $10 \mathrm{~min}$ and then hybridized at $37^{\circ} \mathrm{C}$ overnight. The sections were stringently washed with $50 \%$ formaldehyde and $2 \mathrm{X}$ standard saline citrate (SSC) and 1X SSC, and X-chromosome signals were detected using anti-Dig-Cy3 (Chromosome Science Labo, Inc.). The sections were counterstained with DAPI (Merck KGaA) and mounted with an antifade mounting medium (MountMed; Chromosome Science Labo, Inc.). A CW-4000 cytogenetic workstation (Leica Microsystems, Inc.) was used to detect the FISH signals and analyze the data. An objective lens (magnification, x40) was used for imaging. More than 100 cells were counted for both $\mathrm{X}$ and $\mathrm{Y}$ signals in one slide.

Microdissection PCR. Approximately $2010-\mu \mathrm{m}$ frozen sections were prepared from a frozen specimen, and laser capture microdissection was performed (Fig. 1A) to collect cancer cells with as little mixed normal epithelia, hemocytes or stromal tissues as possible. DNA was extracted using a QIAamp Fast DNA Tissue kit (Qiagen AB). Amelogenin genes in both $\mathrm{X}$ and $\mathrm{Y}$ chromosomes (AMELX and AMELY) were simultaneously examined by PCR using the following primers: 5'-CCCTGGGCTCTGTAAAGAATAGTG-3' forward and 5'-ATCAGAGCTTAAACTGGGAAGCTG-3' reverse (29). PCR products were separated in a polyacrylamide gel, and sex chromosome patterns were analyzed based on the different sizes of amplified bands. Sequences of 106 and 112 bp were amplified from $\mathrm{X}$ and $\mathrm{Y}$ chromosomes, respectively.

Microarray analysis. Microarray analysis was performed as follows. Total RNA was extracted by lysing the tissues with Isogen (Nippon Gene) following homogenization with a TissueLyser (Qiagen AB). Total RNA (500 ng) was used to generate biotin-labeled cRNA using a GeneChip ${ }^{\circledR}$ 3' IVT $^{\prime}$ PLUS Reagent kit (Thermo Fisher Scientific, Inc.). The biotin-labeled RNA was hybridized to Human Genome U-219 Array Strips (Thermo Fisher Scientific, Inc.). After washing and staining the strips, the signal was developed and scanned using GeneAtlas (Thermo Fisher Scientific, Inc.). Data analysis was conducted using GeneSpring GX 14.9.1 


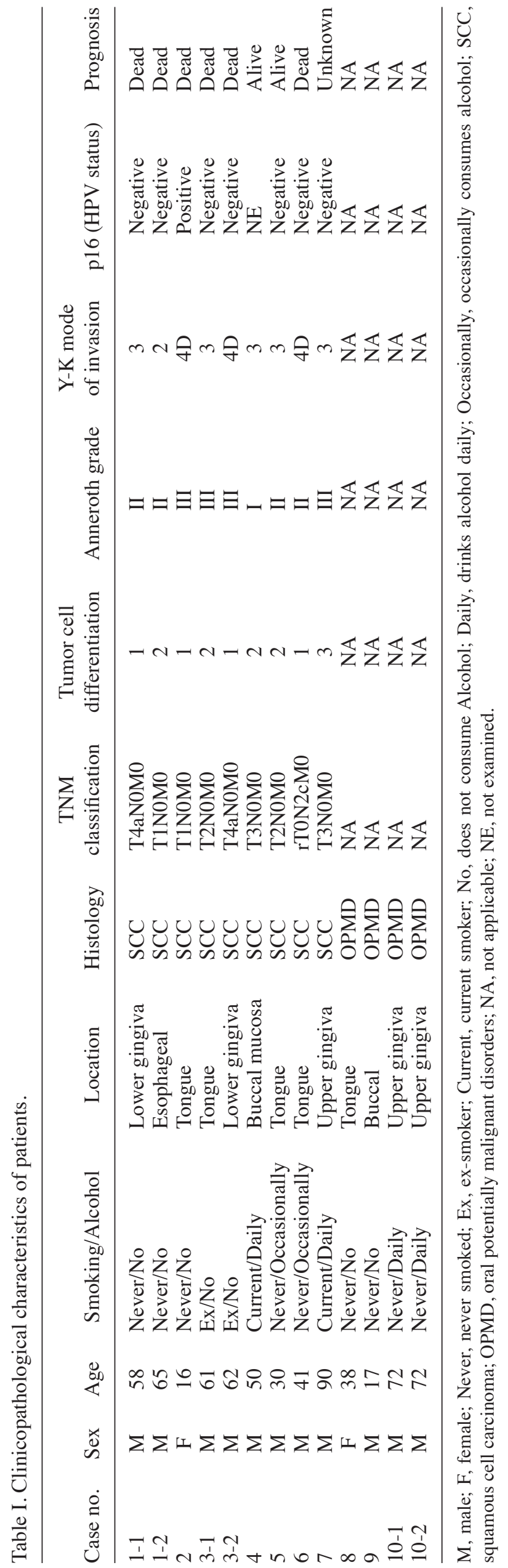

(Agilent Technologies $\mathrm{GmbH}$ ). The robust multichip average method was used with background correction and normalization. Fold change analysis was performed to identify genes with $>3$-fold differences, using a moderated t-test $(\mathrm{P}<0.05)$ with Benjamini-Hochberg multiple correction. Gene expressions of KRT8, ABCC3, GCLC, RYBP, TMEM97, SLC1A3, IRS2, KYNU, CSAG2, CDA and CCL21 in head and neck SCCs (528 cases) from the cancer genome atlas were analyzed by OncoPrint from cBioPortal. Microarray data have been deposited in the Gene Expression Omnibus (GEO; experiment. no. GSE153918) database using minimum information about microarray experiment guidelines. The algorithm of the clustering method for the microarray data is shown in Fig. S1.

\section{Results}

Sex chromosome analysis of OSCC following HSCT. In three male transplanted patients (cases 1, 3 and 4), more X-only cells were identified than Y-containing cells in the tissue (Table II), suggesting the presence of donor-derived cells in OSCC (case 3-1; Fig. 1A). In a second primary cancer that developed in the gingiva of case 3 (case 3-2), a small number of cancer cells were found in the FISH specimen, but most were normal epithelial cells. However, only $\sim 6 \%$ were X-only cells; almost all cancer cells within the observable FISH range were X-only, again indicating donor-derived cells in OSCC. Distant metastasis was observed in $2 / 3$ patients, resulting in a poor prognosis.

Oral cancer tissues in 1 female (case 2) and 2 males (cases 5 and 6) transplanted patients contained cells with sex chromosomes matching the sex of the recipient (Table II), suggesting that OSCC derived from recipient cells (case 5; Fig. 1B). In oral cancer tissues from 1 male patient (case 7), who did not undergo HSCT, there were $13 \% \mathrm{X}$-only cells and $87 \%$ Y-containing cells. In oral potentially malignant disorder (OPMD) epithelial cells from a female patient (case 8) who underwent HSCT, there were $10.7 \%$ Y-containing cells, suggesting that some epithelial cells were from the donor (Fig. 1C). Adjacent normal-appearing oral squamous tissues were compared to oral SCC or OPMD tissues in 5 cases. Some normal-appearing oral squamous cells (3.7-19.8\%) were repaired by donor BM stem cells (case 3-1; Fig. 1D). In normal oral mucosa tissues from two male patients (cases 11 and 12) who did not undergo HSCT, there were $99.1 \%$ (case 11; Fig. 1E) and $100 \%$ Y-containing cells, respectively. Most cells in patients with OSCC had hypertetraploid patterns, including XXXY, XXXYY and XXYYY.

Sex chromosome analysis in OSCC by microdissection PCR. Blood from 1 male patient (case 1) showed an X-only pattern in G-band staining; OSCC cells also showed the same pattern, suggesting that tumor cells were derived from the donor (30). The chromosome patterns in OSCC cells and normal squamous cells were confirmed using laser-captured microdissection PCR with AMELX and AMELY gene amplification (Fig. 2A and B). The results of microdissection PCR showed that OSCC tissues (lane 8) in a male patient (case 1) and cancer tissues following LCM (lane 9) had an X-dominant pattern; therefore, these cells were considered to be derived from the donor (Fig. 2C). 


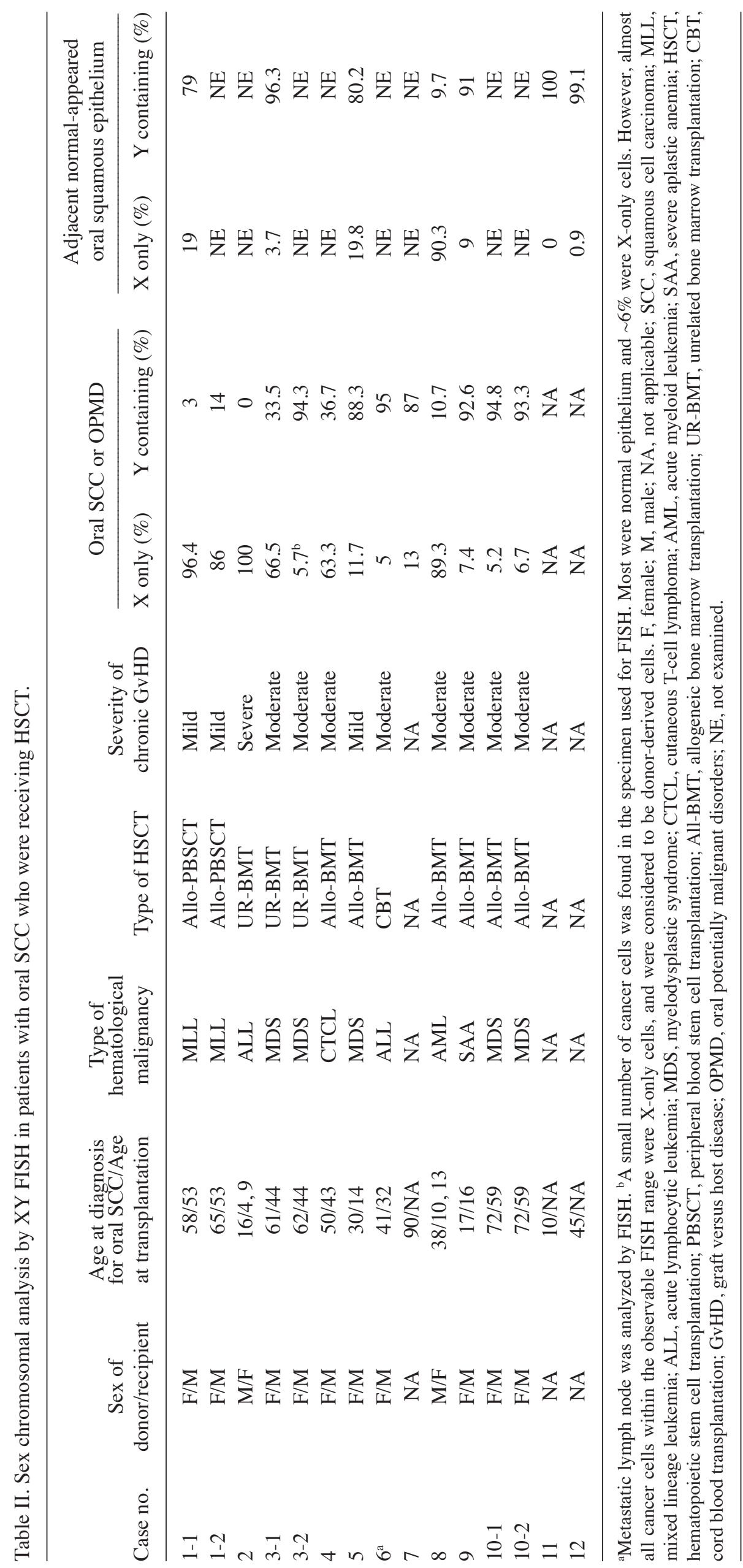




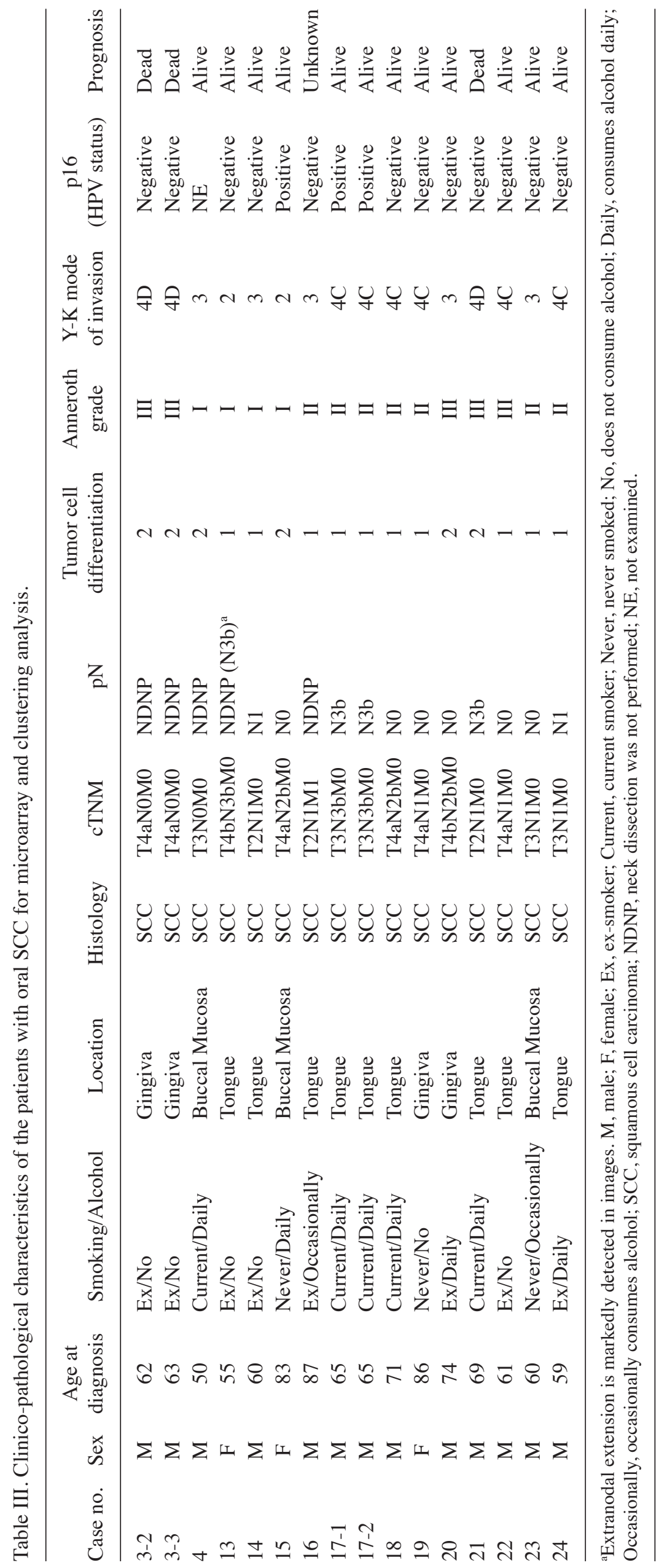



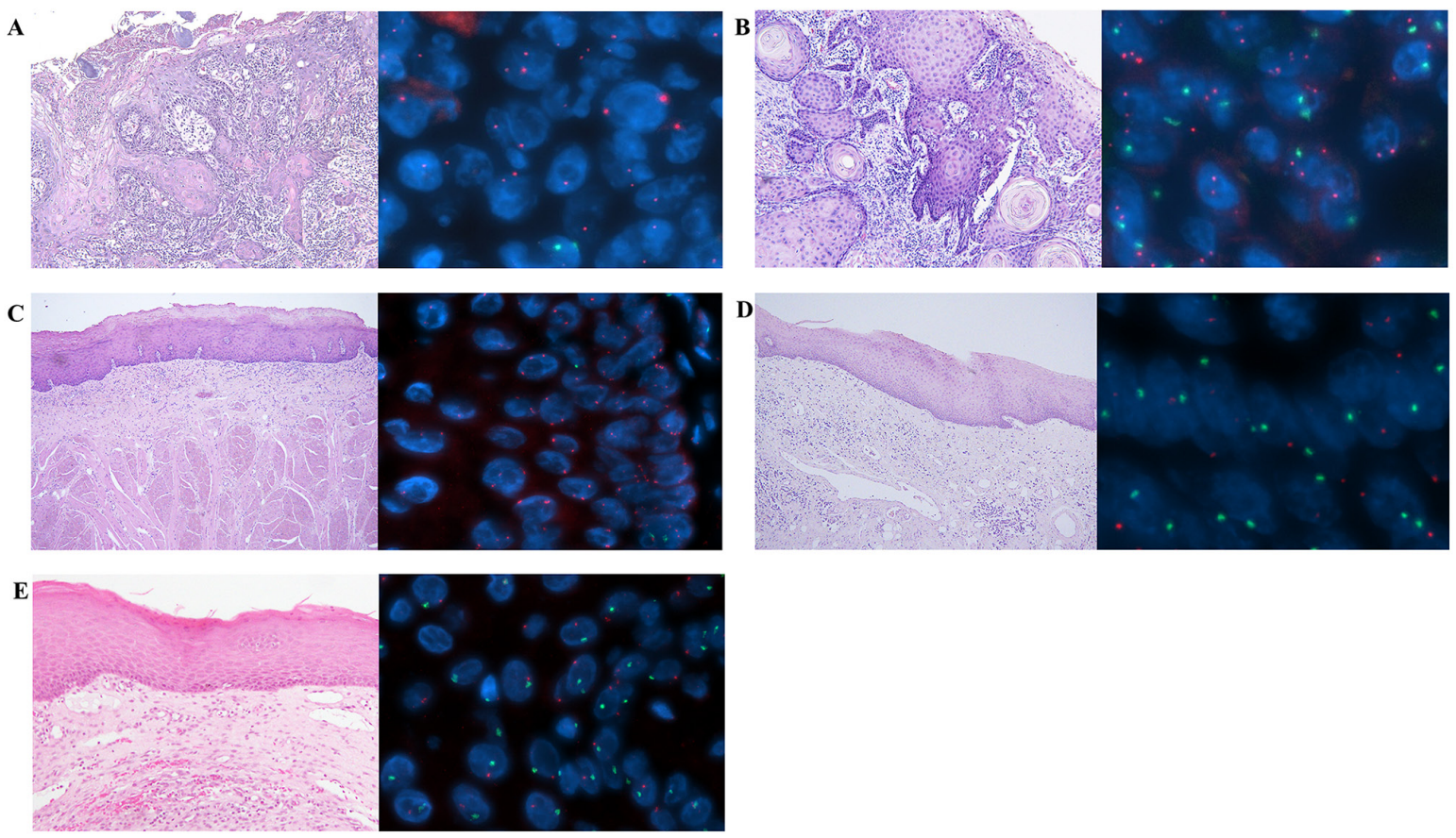

Figure 1. Fluorescence in situ hybridization images of sex chromosomes. (A) A male patient who developed OSCC following HSCT from a female donor OSCC contained of $66.5 \%$ X-only cells and 33.5\% Y-containing cells, indicating that it was donor-derived. (B) A male patient who developed OSCC following HSCT from a female donor. OSCC contained of $19.8 \%$ X-only cells and $80.2 \%$ Y-containing cells, indicating a recipient-derived tumor. (C) A female patient with an oral potentially malignant disorder of the oral mucosa following HSCT from a male donor. The sample contained of $89.3 \%$ X-only cells and $10.7 \%$ Y-containing cells, indicating partial epithelia restoration by transplanted bone marrow cells. (D) A male patient who developed OSCC following HSCT from a female donor. Normal-appearing oral squamous cells contained of 3.7\% X-only cells and 96.3\% Y-containing cells, indicating partial epithelia restoration by transplanted bone marrow cells. (E) Normal labial mucosa in a male with 100\% Y-containing cells. Magnification for hematoxylin-eosin staining, x40; Magnification of fluorescence in situ hybridization images, $\mathrm{x} 400$. OSCC, oral squamous cell carcinoma; HSCT, hematopoietic stem cell transplantation.

Microarray and cluster analysis. The gene expression patterns of patients with possibly BM stem cell-derived OSCC ( 3 samples; cases 3 and 4) were compared to those of patients with normally developed OSCC (13 samples; cases 13-24). The expression patterns in each group were evidently different (Fig. 3). In 3 samples of possibly BM stem cell-derived OSCC, high expression levels ( $>3$-fold on average) were found for KRT8, ABCC3, GCLC, RYBP, TMEM97, SLC1A3, and IRS2, and low expression levels ( $<3$-fold) were found for KYNU, CSAG2///CSGA3, CDA, and CCL21. Cluster analysis based on the signal intensity detected by probes in 14 OSCC patients (16 samples) identified a cluster in 5 samples including possibly BM stem cell-derived OSCCs (3 samples, case 3-2, 3-3 and 4; Fig. 3). Two patients (cases 13 and 20) were included in the cluster in addition to 3 samples from 2 patients (case 3 and 4 ) used for selecting probes. These patients (case 13 and 20) had extremely aggressive tumors: Locally advanced T4b, multiple cervical lymph node metastases and extranodal extension N3b, or locally advanced T4b. The expressions of the marker genes identified in this experiment were examined in head and neck SCC (528 cases) from the cancer genome atlas (Fig. S2). The alterations of the individual gene expression were very limited in each case, and no statistical correlation was identified between the expression levels of these marker genes and prognosis.

\section{Discussion}

A therapeutic strategy based on the assessment of tumor grade has previously been used on factors such as histopathological features, radiographic images, gene mutation and gene expression. OSCC was routinely assessed by TNM staging, histopathological grade, HPV status, and analysis of p53 and relevant molecular abnormalities (11). In a recent study, it was found that some SCCs derived from salivary gland stem cells were found in patients with OSCC, significantly affecting prognosis and the true malignancy of tumor cells (29).

In the present study, it was found that OSCC could develop from BM-derived stem cells. OSCC occurring from more undifferentiated stem cells, such as BM-derived stem cells, might share the characteristics of stem cells (stemness is characterized by invasion of surrounding tissues, viability of vessels and ectopic sites, and self-replicating ability); consequently, such cases had a high malignancy and poor prognosis. A total of 2/3 patients (cases 1 and 3) who developed donor-derived OSCC following HSCT died from multiple distant metastases, and the other patient (case 4) had superficial cancer and was being carefully followed up, although only the 1-year prognosis was good at the time. The two patients (cases 13 and 20) with OSCC possibly derived from autologous BM stem cells exhibited significant cervical metastases that were controlled by extended surgery and intensive chemotherapy. These patients were also under strict follow up.

In the present study, no statistical differences in clinicopathological conditions and prognosis between OSCC from possibly BM-derived stem cells and normally developed OSCC were observed, due to the small number of 


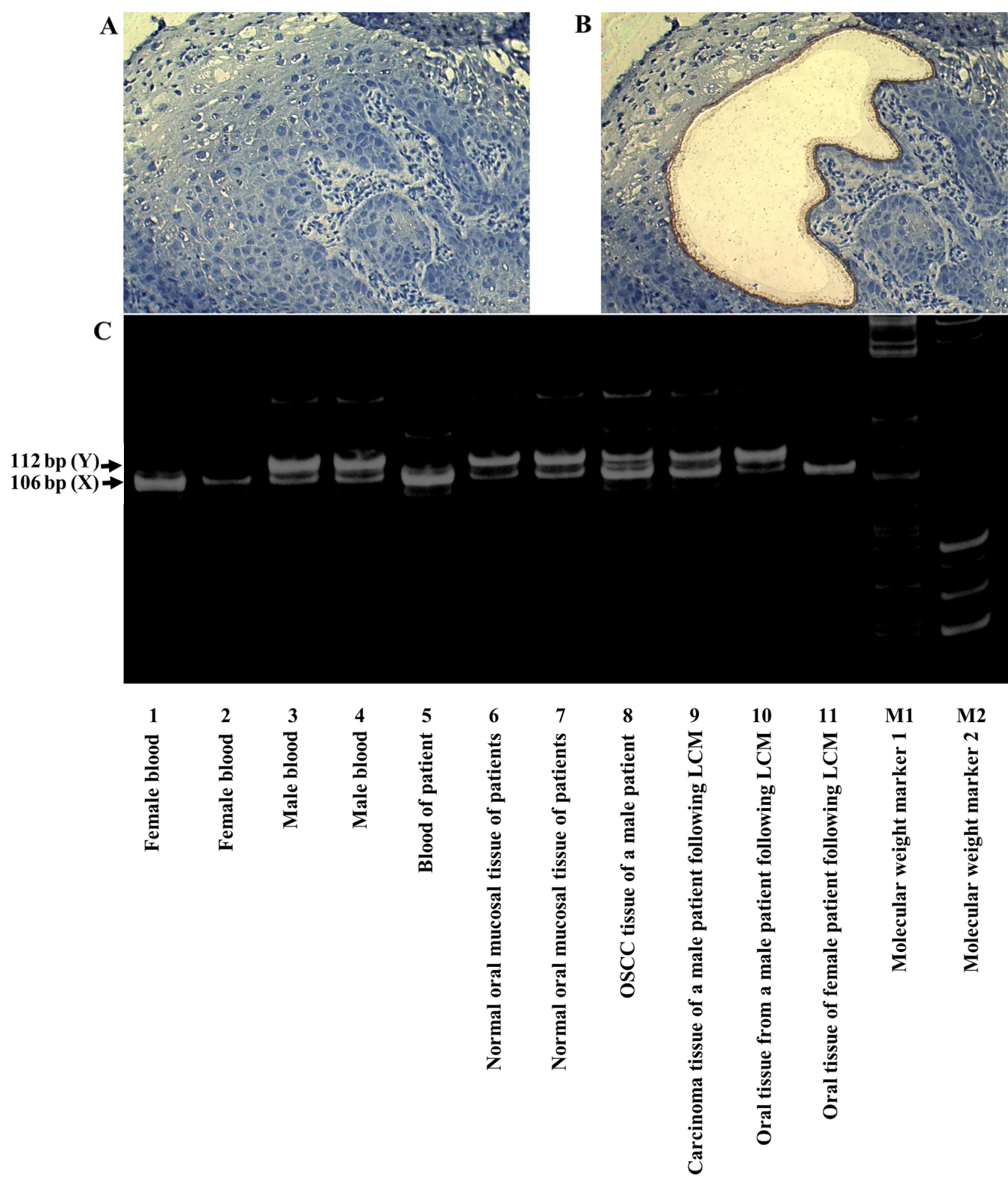

Figure 2. X- and Y-chromosomes in tumor tissues identified by microdissection PCR. (A) Before cancer cell processing by LCM (magnification x200). (B) After cancer cell processing by LCM (magnification, x200). (C) PCR amplification of Amelogenin genes. Lanes 1 and 2, female blood; lanes 3 and 4 , male blood; 5 , blood of patient; 6 and 7, normal oral mucosal tissue of patients; 8 , OSCC tissue of a male patient; 9 , carcinoma tissue of a male patient following LCM; 10, oral tissue from a male patient following LCM; 11, oral tissue of female patient following LCM; M1, molecular weight marker 1; M2, molecular weight marker 2. OSCC (lane 8) and cancer tissues following LCM (lane 9) exhibited an X-dominant pattern; therefore, these were considered to be donor-derived cells. LCM, laser captured microdissection; OSCC, oral squamous cell carcinoma.

cases examined (Kaplan-Meier analysis; data not shown). Therefore, an attempt was made to access the expression patterns of genes with $>3$-fold changes in OSCC from possibly BM-derived stem cells in 167 OSCC samples from a public database (GEO; https://www.ncbi.nlm.nih.gov/geo, experiment number, GSE30784), and cluster analysis was performed. However, no marked cluster could be found. An attempt was also made to extract cases from the public database that had similar expression patterns to those of our 4 patients (5 samples; case 3-2, 3-3, 4, 13 and 20) with possibly $\mathrm{BM}$ stem cell-derived OSCC. However, this was also unsuccessful, due to the different probe types and analysis software used. The accumulation of more data from our microarray analysis is ongoing, in order to develop a method to identify patients with possibly BM stem cell-derived OSCC among patients with normally developed OSCC, and to analyze the clinical characteristics of the possibly BM stem cell-derived OSCC. Separately, a cluster analysis with a combination of ES cell-maintaining markers ( 24 genes) was performed in our OSCC patients or those from the public database; however, no cluster was found. The results showed that genes maintaining ES phenotype (general stemness-maintaining genes) were highly expressed in almost all OSCCs, indicating the reversion of cancer cells to stem cells.

As shown by XY-FISH analysis conducted in a very small population of the cells, a Y-chromosome signal was not detectable in male patients with OSCC who did not undergo HSCT. This may be due to technical errors of FISH or Y chromosome dropout 

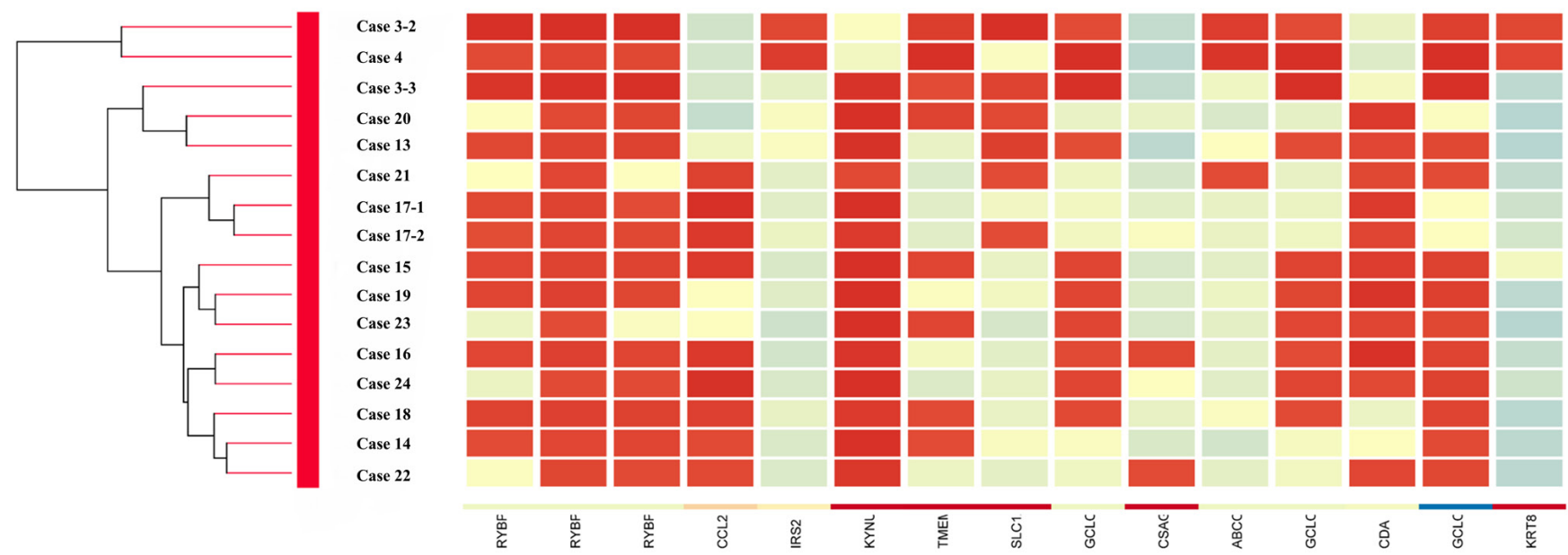

Figure 3. Clustering analysis from microarray data. Clustering was performed in 16 OSCC samples (14 patients) using genes with high (KRT8, ABCC3, GCLC, RYBP, TMEM97, SLC1A3 and IRS2) and low (KYNU, CSAG2///CSGA3, CDA and CCL21) expression levels in 3 samples of donor bone marrow-derived stem cells determined by sex chromosome analysis. Red indicates high expression and blue indicates low expression. A cluster was formed in 5 samples (cases 3-2, 3-3, 4, 13 and 20). OSCC, oral squamous cell carcinoma.

during malignant transformation. However, OSCC from male patients who underwent HSCT exhibited X-only signal in the majority of the cells, which might suggest that malignant transformation occurred in the epithelial cells from female donors. Furthermore, some epithelial cells in OPMDs of female patients contained Y chromosomes, which showed that a portion of the epithelial cells in the lesion might have been derived from BM stem cells of the male donor. These results suggest that a portion of the epithelial cells in the oral mucosa were repaired using donor BM stem cells in several patients following HSCT, possibly resulting in malignant transformation to SCC. A total of $2 / 3$ (cases 13 and 20) patients with OSCC derived from donor BM stem cells developed multiple cancer, so-called 'field cancerization', in the upper digestive tract, where chronic GvHD occurred and epithelial cells were repaired in BM-derived stem cells. Although the influence of chronic inflammation and immune-suppressants appeared to be important for oral carcinogenesis following HSCT, a chronic persistent inflammation-regeneration (by BM-derived stem cells)-metaplasia-carcinoma sequence occurs in the oral mucosa. To the best of our knowledge, this is the first report that focuses on oral squamous carcinogenesis. Kano et al examined patients with esophageal SCC that developed from BM-derived stem cells and found similar results (33). In order to prove a chronic persistent inflammation-regeneration by BM derived stem cells-metaplasia-carcinoma sequence in the oral SCC patients who underwent HSCT, a multi-institutional big study might be necessary.

BM-derived stem cells consist of hematopoietic and mesenchymal stem cells, the latter of which can differentiate into different types of epithelial cells. Among these, Muse cells have been widely studied and found in the blood, as well as in stromal tissue from the whole body, leading to differentiation into tridermic cells. In contrast to ES and iPS cells, Muse cells were considered as pluripotent stem cells without a potential for neoplastic transformation (28). The population of the BM-derived stem cells that lead to OSCC is unclear; however, it is likely that it is a population of non-Muse mesenchymal stem cells. We are currently conducting an animal study to identify the subpopulation of BM-derived stem cells that can repair the oral epithelium and may lead to the development of OSCC. To the best of our knowledge, any animal models of oral SCC carcinogenesis which supported our hypothesis had not been reported. We recently established the BM stem cells transplantation model in mice. We will plan to inoculate the sorted sub-population of the GFP labeled-BM cells to the recipient mice. Then, an oral carcinogenesis experiment, a chronic persistent inflammation-regeneration-metaplasia-carcinoma, will be performed in the recipient mice.

The conventional therapeutic strategy for OSCC was made according to the clinical and pathological findings, radiographic features, mutations and expressions of a limited number of genes, regardless of the origin of the tumor. OSCC might consist of the carcinomas derived from epithelial stem cells, salivary gland stem cells, and BM-derived stem cells. If the genes that can discriminate the origin are identified, OSCC could be classified into three categories based on cell origin, but not superficial phenotype. An origin-based diagnosis of cancer cells can help develop a more specific therapeutic strategy to improve prognosis. This would be a paradigm shift in the diagnostic and therapeutic strategies for OSCC.

\section{Acknowledgments}

The authors would like to thank Professor Kinuko Mitani (Department of Hematology and Oncology, Dokkyo Medical University School of Medicine), Professor Shigemi Yoshihara (Department of Pediatrics, Dokkyo Medical University School of Medicine), Professor Keiichi Kubota (Department of Gastroenterological Surgery, Dokkyo Medical University School of Medicine) and Professor Kazuyuki Ishida (Department of Molecular Diagnostic Pathology, Dokkyo Medical University School of Medicine) for providing the clinical and pathological information of the patients. The authors would also like to thank Ms. Chiaki Matsuyama, Ms. Ayako Shimizu (Department of Molecular Diagnostic Pathology, Dokkyo Medical University School of Medicine) and Mr. Kazumi Akimoto (Clinical Research Center, Dokkyo Medical University School of Medicine) for their technical assistance. 


\section{Funding}

This study was supported by the Young Investigator Award of Dokkyo Medical University (grant no. 2018-16, 2019-14) and Japan Society for the Promotion of Science Grant-in-Aid for Scientific Research [grant no. (C) (17K11887)].

\section{Availability of data and material}

The datasets during and/or analyzed during the current study available from the corresponding author on reasonable request.

\section{Authors' contributions}

THa, KIN, CF, THy, YS, MS, RK, NK, AF, SU and HK contributed to the study conception and design. THa, THy, YS, MS, RK and NK collected the data. THa, CF, AF, KIN, DU and HK analyzed the data. THa drafted the manuscript. THa, KIN, CF, THy, YS, MS, RK, NK, AF, SU and HK reviewed the final draft of the manuscript. All authors read and approved the final manuscript.

\section{Ethics approval and consent to participate}

The design of the current study was approved by the Medical Ethical Research Committee of Dokkyo Medical University Hospital (approval ID, R-25-16J) and Ehime University (approval ID, 2007024). Written informed consent was obtained from all patients for the use of their samples for research and for publication at the start of the treatment. The patients could also opt-out, based on a research plan approved by the Medical Ethical Research Committee of our institutes. We confirm that no opt-outs were recorded.

\section{Patient consent for publication}

Not applicable.

\section{Competing interests}

The authors declare that they have no competing interests.

\section{References}

1. Johnson N, Franceschi S, Ferlay J, Ramadas K, Schmid S, MacDonald DG, Bouquot JE and Slootweg PJ: Squamous cell carcinoma. In: Pathology and Genetics of Head and Neck Tumours. Barnes L, Eveson JW, Reichart P, Sidransky D (eds.) World Health Organization classification of tumours, 1st edition. IARCPress, Lyon: 168-175, 2005.

2. Shah JP, Zelefsky MJ and O'Malley BB: Squamous cell carcinoma of the oral cavity. In: Head and Neck Cancer. Harrison LB, Sessions RB, Hong WK (eds.) Lippincott-Raven, New York, USA, pp 411-418, 1999.

3. Ginos MA, Page GP, Michalowicz BS, Patel KJ, Volker SE, Pambuccian SE, Ondrey FG, Adams GL and Gaffney PM: Identification of a gene expression signature associated with recurrent disease in squamous cell carcinoma of the head and neck. Cancer Res 64: 55-63, 2004.

4. Uchida D, Begum NM, Almofti A, Nakashiro Ki, Kawamata H, Tateishi Y, Hamakawa H, Yoshida H and Sato M: Possible role of stromal-cell-derived factor-1/CXCR4 signaling on lymph node metastasis of oral squamous cell carcinoma. Exp Cell Res 290: 289-302,2003.

5. Langer CJ: Exploring biomarkers in head and neck cancer. Cancer 118: 3882-3892, 2012.
6. Sheikh Ali MA, Gunduz M, Nagatsuka H, Gunduz E, Cengiz B, Fukushima K, Beder LB, Demircan K, Fujii M, Yamanaka N, et al: Expression and mutation analysis of epidermal growth factor receptor in head and neck squamous cell carcinoma. Cancer Sci 99: 1589-1594, 2008.

7. Ang KK, Berkey BA, Tu X, Zhang HZ, Katz R, Hammond EH, $\mathrm{Fu}$ KK and Milas L: Impact of epidermal growth factor receptor expression on survival and pattern of relapse in patients with advanced head and neck carcinoma. Cancer Res 62: 7350-7356, 2002.

8. Sheu JJ, Hua CH, Wan L, Lin YJ, Lai MT, Tseng HS, Jinawath N, Tsai MH, Chang NW, Lin CF, et al: Functional genomic analysis identified epidermal growth factor receptor activation as the most common genetic event in oral squamous cell carcinoma. Cancer Res 69: 2568-2576, 2009.

9. Azuma M, Yoshida H, Kawamata H, Yanagawa T, Furumoto N and Sato M: Cellular proliferation and ras oncogene of p21 21,000 expression in relation to the intracellular cyclic adenosine 3': 5'-Monophosphate levels of a human salivary gland adenocarcinoma cell line in culture. Cancer Res 48: 2898-2903, 1988.

10. Shinagawa Y, Kawamata H, Omotehara F, Nakashiro Ki, Hoque MO, Furihata T, Horiuchi H, Imai Y, Fujimori T and Fujibayashi T: Evaluation of the chemosensitivity of head and neck cancer cells based on the diverse function of mutated-p53. Int J Oncol 22: 383-389, 2003.

11. Tachibana M, Shinagawa $\mathrm{Y}$, Kawamata H, Omotehara F, Horiuchi H, Ohkura Y, Kubota K, Imai Y, Fujibayashi T and Fujimori T: RT-PCR amplification of RNA extracted from formalin-fixed, paraffin-embedded oral cancer sections: Analysis of p53 pathway. Anticancer Res 23: 2891-2896, 2003.

12. Hoque MO, Kawamata H, Nakashiro KI, Omotehara F, Hino S, Uchida D, Harada K, Begum NM, Yoshida H, Sato M and Fujimori T: Dysfunction of the p53 tumor suppressor pathway in head and neck cancer. Int J Oncol 21: 119-126, 2002.

13. Nagata M, Noman AA, Suzuki K, Kurita H, Ohnishi M, Ohyama T, Kitamura N, Kobayashi T, Uematsu K, Takahashi K, et al: ITGA3 and ITGB4 expression biomarkers estimate the risks of locoregional and hematogenous dissemination of oral squamous cell carcinoma. BMC Cancer 13: 410, 2013.

14. Kashyap T, Germain E, Roche M, Lyle S and Rabinovitz I: Role of $\beta 4$ integrin phosphorylation in human invasive squamous cell carcinoma: Regulation of hemidesmosome stability modulates cell migration. Lab Invest 91: 1414-1426, 2011.

15. Kurokawa A, Nagata M, Kitamura N, Noman AA, Ohnishi M, Ohyama T, Kobayashi T, Shingaki S, Takagi R; Oral, Maxillofacial Pathology, and Surgery Group: Diagnostic value of integrin alpha3, beta4, and beta5 gene expression levels for the clinical outcome of tongue squamous cell carcinoma. Cancer 112: 1272-1281, 2008

16. Mitra RS, Goto M, Lee JS, Maldonado D, Taylor JM, Pan Q, Carey TE, Bradford CR, Prince ME, Cordell KG, et al: Rap1GAP promotes invasion via induction of matrix metalloproteinase 9 secretion, which is associated with poor survival in low N-stage squamous cell carcinoma. Cancer Res 68: 3959-3969, 2008.

17. Kawamata $H$, Uchida $D$, Hamano $H$, Kimura-Yanagawa $T$, Nakashiro KI, Hino S, Omotehara F, Yoshida $\mathrm{H}$ and Sato M: Active-MMP2 in cancer cell nests of oral cancer patients: Correlation with lymph node metastasis. Int J Oncol 13: 699-704, 1998.

18. Kawamata H, Nakashiro K, Uchida D, Harada K, Yoshida H and Sato M: Possible contribution of active MMP2 to lymphnode metastasis and secreted cathepsin $\mathrm{L}$ to bone invasion of newly established human oral-squamous-cancer cell lines. Int J Cancer 70: 120-127, 1997.

19. Mineta H, Miura K, Suzuki I, Takebayashi S, Amano H, Araki K, Harada H, Ichimura K, Wennerberg JP and Dictor MR: Low p27 expression correlates with poor prognosis for patients with oral tongue squamous cell carcinoma. Cancer 85: 1011-1017, 1999.

20. Kanazawa T, Iwashita T, Kommareddi P, Nair T, Misawa K, Misawa Y, Ueda Y, Tono T and Carey TE: Galanin and galanin receptor type 1 suppress proliferation in squamous carcinoma cells: Activation of the extracellular signal regulated kinase pathway and induction of cyclindependent kinase inhibitors. Oncogene 26: 5762-5771, 2007.

21. Supriatno, Harada K, Yoshida $\mathrm{H}$ and Sato M: Basic investigation on the development of molecular targeting therapy against cyclin-dependent kinase inhibitor p27Kip1 in head and neck cancer cells. Int J Oncol 27: 627-635, 2005.

22. Yamamoto E, Kohama G, Sunakawa H, Iwai M and Hiratsuka H: Mode of invasion, bleomycin sensitivity and clinical course in squamous cell carcinoma of the oral cavity. Cancer 51: 2175-2180, 1983. 
23. Alison MR, Poulsom R, Jeffery R, Dhillon AP, Quaglia A, Jacob J, Novelli M, Prentice G, Williamson J and Wright NA: Hepatocytes from non-hepatic adult stem cells. Nature 406: 257, 2000.

24. Jackson KA, Majka SM, Wang H, Pocius J, Hartley CJ, Majesky MW, Entman ML, Michael LH, Hirschi KK and Goodell MA: Regeneration of ischemic cardiac muscle and vascular endothelium by adult stem cells. J Clin Invest 107: 1395-1402, 2001.

25. Okamoto R, Yajima T, Yamazaki M, Kanai T, Mukai M, Okamoto S, Ikeda Y, Hibi T, Inazawa J and Watanabe M: Damaged epithelia regenerated by bone marrow-derived cells in the human gastrointestinal tract. Nat Med 8: 1011-1017, 2002.

26. Houghton J, Stoicov C, Nomura S, Rogers AB, Carlson J, Li H, Cai X, Fox JG, Goldenring JR and Wang TC: Gastric cancer originating from bone marrow-derived cells. Science 306: 1568-1571, 2004.

27. Tamai K, Yamazaki T, Chino $\mathrm{T}$, Ishii M, Otsuru S, Kikuchi Y, Iinuma S, Saga K, Nimura K, Shimbo T, et al: PDGFRalpha-Positive cells in bone marrow are mobilized by high mobility group box 1 (HMGB1) to regenerate injured epithelia. Proc Natl Acad Sci USA 108: 6609-6614, 2011.

28. Kuroda Y, Kitada M, Wakao S, Nishikawa K, Tanimura Y, Makinoshima $\mathrm{H}$, Goda M, Akashi H, Inutsuka A, Niwa A, et al: Unique multipotent cells in adult human mesenchymal cell populations. Proc Natl Acad Sci USA 107: 8639-8643, 2010
29. Kinouchi M, Izumi S, Nakashiro K, Haruyama Y, Kobashi G, Uchida D, Hasegawa T and Kawamata H: Determination of the origin of oral squamous cell carcinoma by microarray analysis: Squamous epithelium or minor salivary gland?. Int J Cancer 143: 2551-2560, 2018

30. Arai Y, Arai H, Aoyagi A, Yamagata T, Mitani K, Kubota K, Kawamata $\mathrm{H}$ and Imai $\mathrm{Y}$ : A solid tumor of donor cell-origin after allogeneic peripheral blood stem cell transplantation: Am J Transplant 6: 3042-3043, 2006.

31. Pindborg JJ, Reichart PA, Smith CJ and van der Waal I: Histological Typing of Cancer and Precancer of the Oral Mucosa, 2nd edition. Springer, Berlin, 1997.

32. Anneroth G, Batsakis J and Luna M: Review of the literature and a recommended system of malignancy grading in oral squamous cell carcinomas. Scand J Dent Res 95: 229-249, 1987.

33. Kano Y, Ishii H, Konno M, Yamasaki M, Miyata H, Nishikawa S, Hamabe A, Ogawa H, Takahashi H, Ohta K, et al: Cells of origin of squamous epithelium, dysplasia and cancer in the head and neck region after bone marrow transplantation. Int J Oncol 44: 443-450, 2014

(c) (9) $\Theta$ This work is licensed under a Creative Commons Attribution-NonCommercial-NoDerivatives 4.0 International (CC BY-NC-ND 4.0) License. 